\title{
Zur Normalisierung heteronormativer Zweigeschlechtlichkeit im Recht: Eine queere Analyse der Regulation des Geschlechtswechsels im Vereinigten Königreich ${ }^{1}$
}

Dieser Beitrag problematisiert unter Verwendung des analytischen Instrumentariums der queer theory das Recht als einen der Machtapparate im Konstruktionsprozess des historisch-spezifischen und dynamischen Regimes heteronormativer Zweigeschlechtlichkeit. Dies geschieht am Beispiel des Gender Recognition Act (GRA), der Verschiebungen im Geschlechterdiskurs widerspiegelt, die sich auch in der anhaltenden Debatte um das noch zu reformierende deutsche Transsexuellengesetz (TSG) andeuten.

Am I. Juli 2004 trat der GRA im Vereinigten Königreich in Kraft. Das Gesetz ermöglicht es, das Geschlecht von Personen anzuerkennen, die sich dem anderen der zwei legitimierten Geschlechter zugehörig fühlen. Ihm waren jahrzehntelange Anstrengungen individueller Transpersonen ${ }^{2}$ auf nationaler ${ }^{3}$ und internationaler ${ }^{4}$ rechtlicher Ebene sowie Auseinandersetzungen von Transorganisationen' auf dem innenpolitischen Terrain im Vorfeld und während des Gesetzgebungsprozesses vorangegangen. ${ }^{6}$

Der GRA entstand in dem Kontext der Verschiebung des Transdiskurses von vergleichsweise rigiden und homogenen Konstruktionen des transsexuellen Subjektes zu einer Individualisierung und Pluralisierung von Subjektpositionen.7 Im medizinisch-psychiatrischen Bereich spiegeln sich diese Verschiebungen in der Flexibilisierung und Individualisierung des Behandlungskonzepts von transsexuellen Individuen wider. ${ }^{8}$ In der Transbewegung manifestieren sie sich

I In Erinnerung an Harm Dunkhase.

Ich danke Juana Remus und Laura Adamietz für zahlreiche rechtswissenschaftliche Literaturhinweise sowie Nora Markard, Corinna Bath, Sonja Buckel, Andreas Fischer-Lescano und Felix Hanschmann für hilfreiche Kommentare zum Manuskript.

2 Ich bevorzuge die Begriffe »Transpersonen « oder »transgeschlechtliche Individuen«, weil sie für eine Vielzahl von Personen stehen, deren Geschlechtsidentitäten nicht der vermeintlichen Kongruenz von Morphologie und Geschlechtsidentität entsprechen und ich sie zudem als in einem gleichrangigen Verhältnis zu anderen Geschlechtern stehend betrachte. Beziehe ich mich auf konkrete Texte, übernehme ich die Begriffe oder Attribute in Bezug auf das Phänomen, wie sie die jeweiligen Verfasser_innen verwenden, um ihre jeweiligen Aussagen nicht zu verfälschen.

3 Vgl. Corbett v. Corbett (Otherwise Ashley), [1970] 3 W.L.R. I95; Bellinger v. Bellinger, [2003] UKHL 2 I.

4 EGMR, Urt. v. 17.10.1986, Rees v. UK [1986] 9 EHRR 56; EGMR, Urt. v. 27.9.1990, Cossey v. UK [1990] I 3 EHHR 622; EGMR, Urt. v. I1.7.2002, Goodwin v. UK [2002] 35 EHRR I 8.

5 Allen voran ist in diesem Zusammenhang die 1992 gegründete Interessengruppe Press For Change zu nennen. S. www.pfc.org.uk.

6 Diese und weitere Ausführungen zum Gesetzgebungsprozess s. Adrian de Silva, Zur Konstruktion von Geschlecht und Geschlechterregimen in dem Gender Recognition Act 2004 und im englischen Parlament, in: liminalis I (2007), 83-108, [www.liminalis.de/artikel/Liminalis2007_desilva.pdf].

7 Auch die Urteilsbegründung des Bundesverfassungsgerichts zum $\$ 7$ Abs. I Nr. 3 TSG vom 6.I 2.2005 spiegelt diese Diskursverschiebung wider. Vgl. BVerfGE I I s, I. Zu den Vorgängen, die hierzu führten, s. Laura Adamietz, Transgender ante portas? Anmerkungen zur fünften Entscheidung des Bundesverfassungsgerichts zur Transsexualität, Kritische Justiz 39/2006, 368-380.

8 So sieht die sechste Version der Harry Benjamin Standards of Care z.B. vor, Hormongaben zur Angleichung an das gelebte Geschlecht auch dann zuzulassen, wenn ein Individuum ggf. chirurgische Maßnahmen ablehnt. Vgl. Walter Meyer et al., The Harry Benjamin International Gender Dysphoria Association's Standards of Care For Gender Identity Disorders. Sixth Version, 200I, I-22, 2, [wpath.org/ Documents2/socv6.pdf]. Die Organisation ist mittlerweile unbenannt worden in «World Professional Association for Transgender Health« (WPATH). 
in der Pluralisierung von Geschlechterverständnissen, in dem unterschiedlichen

Ausmaß medizinischer und chirurgischer Maßnahmen, die Individuen benötigen, um in der Rolle des Wunschgeschlechts leben zu können, und in der Dauer der Geschlechtszugehörigkeit. ${ }^{9}$

Ausgehend von zentralen Axiomen des Butler'schen Konzepts heteronormativer Zweigeschlechtlichkeit untersuche ich, wie der GRA geschlechtliche Möglichkeiten derart kanalisiert, dass er - trotz Verschiebungen im Geschlechterdiskurs in Relation zu älteren Gesetzen, wie etwa dem TSG in der Bundesrepublik Deutschland - ein bipolares, heteronormatives Geschlechterregime fortschreibt.

\section{Heteronormative Zweigeschlechtlichkeit bei Judith Butler}

Mit ihrer Interpretation der Kategorie >Geschlecht< als regulatorische Konstruktion, die Heterosexualität privilegiert, und der Dekonstruktion normativer Geschlechtermodelle leitet Butler einen Paradigmenwechsel im Feminismus ein und legt ein theoretisches Fundament für queer theory. ${ }^{\circ}$ Von besonderer Bedeutung für eine Analyse heteronormativer Zweigeschlechtlichkeit sind hierbei die Theoreme zur Dekonstruktion von sex und gender, zur Herstellung des Zusammenhangs von Geschlecht und Heterosexualität sowie zur Herstellung unintelligibler Geschlechter.

Nach Butler sind Konstruktionen von Geschlecht sowohl in historischer als auch in kulturell vergleichender Perspektive derart verschieden, wenn nicht gar widersprüchlich, dass nicht davon ausgegangen werden kann, dass Geschlecht als kulturelle Deutung (gender) von dem körperlichen Geschlecht (sex) abgeleitet werden kann. Betrachtet man gender in letzter Konsequenz gar als radikal unabhängig von sex, könnte >Mann< und >maskulin < beispielsweise genauso einen weiblichen wie auch einen männlichen Körper bezeichnen und >Frau< und >feminin< einen weiblichen oder männlichen Körper. ${ }^{\text {I I }}$

Ebenso dekonstruiert Butler die Vorstellung von einem vordiskursiven Geschlecht des Körpers. Vorausgesetzt, es gibt eine Geschichte über die Herstellung der Dualität von Geschlechtskörpern oder es lässt sich eine diskursive Produktion scheinbar natürlicher Fakten des körperlichen Geschlechts feststellen, so erweist sich das körperliche Geschlecht ebenfalls als kulturelles Geschlecht. Damit handelt es sich sowohl bei der sozialen als auch bei der körperlichen Zweigeschlechtlichkeit um eine gesellschaftliche Konstruktion. Demnach gilt es, die Apparate zu untersuchen, die Körpergeschlechter herstellen und die sie somit als solche erkennbar machen. ${ }^{\mathrm{I} 2}$

Die Herstellung von gender erfolgt nach Butler performativ. Performativität bezeichnet eine Wiederholung der Normen, mit denen das Subjekt überhaupt als solches wahrnehmbar wird. Gender entsteht als performativer Effekt eines regulatorischen Regimes von Geschlechterunterschieden, in dem Geschlechter unter

9 Vgl. Home Office, Report of the Interdepartmental Working Group on Transsexual People, 2000, I-67, 25 , [www.pfc.org.uk/workgrp/wgtrans.pdf]. Eine ähnliche Entwicklung lässt sich auch in der Transbewegung in der Bundesrepublik Deutschland seit den 9oer Jahren beobachten. Vgl. hierzu Adrian de Silva, Transsexualität im Spannungsfeld juristischer und medizinischer Diskurse, Z Sexualforsch (I 8) 2005, 258-27I, $262 \mathrm{ff}$.

Io Einführend Annamarie Jagose, Queer Theory. An Introduction, New York 1996; Sabine Hark, Queer Studies, in: Christina von Braun/Inge Stephan (Hrsg.), Gender @ Wissen. Ein Handbuch der Gender-Theorien, Köln/Weimar/Wien, 285-303. Rechtswissenschaftliche Einführung in Elisabeth Greif/Eva Schobesberger, Einführung in die Feministische Rechtswissenschaft. Ziele, Methoden, Theorien, 2. Aufl. Linz 2007, 70-106.

I I Judith Butler, Gender Trouble. Feminism and the Subversion of Identity, London/ New York I990, 7.

I 2 Ebd. 
Zwang polarisiert und hierarchisiert werden. ${ }^{13}$ Dabei verdichten sich diese Wiederholungen, so dass sie mit der Zeit als natürlich erscheinen. ${ }^{\mathrm{I}}$

Regulatorische Praktiken der Geschlechterbildung produzieren mit einer einheitlichen Identität eines Geschlechts ein normatives Ideal, in dem lediglich diejenigen Geschlechter als sintelligibel<, d.h. als sinnhaft, und diejenige Sexualität als nicht pervers erscheinen, die eine Kohärenz darstellen zwischen sex, gender, sexueller Praxis und Begehren. Hierbei erfordert und reproduziert die Heterosexualisierung von Begehren die Herstellung unterscheidbarer und asymmetrischer Oppositionen zwischen dem >Femininen $<$ und dem >Maskulinen $<.{ }^{15}$ Die binäre Geschlechterordnung ist daher zugleich auch heteronormativ.

Zudem handelt es sich hierbei um ein komplexes, nicht aufeinander reduzierbares Verhältnis zwischen Formen der Sexualität und Geschlecht. Vielmehr ist gender von multiplen sozialen Verflechtungen durchdrungen, die die heterosexuelle Matrix enthalten und über sie hinausgehen. Somit bestimmen Formen der Sexualität gender nicht einseitig. ${ }^{16}$

Da der Geschlechterdiskurs dem Subjekt vorangeht und dieses erst durch Anrufung entsteht, ${ }^{17}$ ist der Status derjenigen, die den vergeschlechtlichten Normen kultureller Intelligibilität nicht folgen, in Frage gestellt. Diejenigen Individuen, deren Geschlechtsidentität nicht sex und deren Begehren nicht sex oder gender folgen, erscheinen als gestört oder als logische Unmöglichkeiten. Sie zeigen jedoch zugleich die Grenzen und regulatorischen Ziele des kulturellen Regimes auf und ermöglichen konkurrierende und subversive Konzepte von Geschlechterordnungen. ${ }^{18}$

Zusammenfassend zeigt Butler auf, wie bestimmte sexuelle und geschlechtliche Konfigurationen sich selbst reproduzierende hegemoniale Formen annehmen und andere damit undenkbar oder ausgeschlossen werden. Vor diesem theoretischen Hintergrund betrachte ich nunmehr den GRA.

\section{Heteronormative Zweigeschlechtlichkeit im Gender Recognition Act}

Die Bestimmungen des GRA lassen darauf schließen, dass Zweigeschlechtlichkeit die unhinterfragte Hintergrundnorm bildet, vor welcher der Geschlechtswechsel gesetzlich geregelt ist. Dies wird deutlich an der Einschränkung von geschlechtlichen Möglichkeiten. So spezifiziert Art. 9 (I) im Falle eines erfolgreichen Antrags, dass, wenn das >erworbene< Geschlecht das männliche sei, das Geschlecht der Person das eines Mannes wird, und wenn es das des weiblichen Geschlechts sei, wird das Geschlecht der Person zu dem einer Frau. ${ }^{19}$

Damit schließt das Statut implizit Subjekte aus, die den Prinzipien polarisierter Zweigeschlechtlichkeit widersprechen. Das Gesetz sieht keine Subjektposition für Individuen vor, die sich jeglicher Einordnung entziehen. D.h. jedes Indivi-

I 3 Judith Butler, Critically Queer, in: Shane Phelan (Hrsg.), Playing With Fire. Queer Politics, Queer Theories, New York/London I997, I I-29, I7.

I 4 Butler (Fn. I I), 33 .

is Ebd., I7. Es ist auch in sozialhistorischer Perspektive auffällig, dass die Polarisierung der zwei rechtlich anerkannten Geschlechter in westlichen Gesellschaften einherging mit der Konstruktion von Homosexualität als wesenhaftem Charakteristikum eines Individuums. Vgl. hierzu u.a. Jeffrey Weeks, Sex, Politics and Society. The Regulation of Sexuality Since r800, 2. Aufl. London/New York 1989, und Stefan Hirschauer, Die soziale Konstruktion der Transsexualität. Über die Medizin und den Geschlechtswechsel, 2. Aufl. Frankfurt am Main 74 ff. Die Begriffe »homo-«, »hetero-« und »bisexuell« machen ohnehin nur vor dem Hintergrund eines zweigeschlechtlichen Regimes Sinn.

I6 Butler (Fn. I3), 23.

I7 Ebd., I 2.

I 8 Butler (Fn. I I), 3 I; dies., Undoing Gender, New York/London 2004, 2 I 8.

I9 Gender Recognition Act 2004, [www.opsi.gov.uk/acts/en2004/2004 en 33 htm]. 
duum muss als ein Geschlecht erkennbar sein. Ebenso wenig sieht der GRA vor, dass ein Individuum mehreren Geschlechtern angehören kann.

Explizit ausgeschlossen sind Subjekte, die ihre Geschlechtszugehörigkeit als variabel betrachten. So bestimmt das GRA in Art. 2 ( I) (b), dass die antragstellende Person mindestens zwei Jahre lang im serworbenen< Geschlecht gelebt haben muss. Des Weiteren muss gemäß Art. 2 (I) (b) i.V.m. Art. 3 (4) das betreffende Individuum eidesstattlich erklären, mit der Anerkennung des Wunschgeschlechts in diesem bis zum Tode weiterleben zu wollen. ${ }^{20}$

Ebenso suggeriert der GRA eine polarisierte Vorstellung der zwei legitimierten Geschlechter. Dies wird anhand von Art. 20 ersichtlich, der >geschlechtsspezifische Vergehen< regelt. Falls ein Straftatbestand nur von einer Person mit einem bestimmten Geschlecht oder nur gegen eine Person mit einem bestimmten Geschlecht begangen werden kann, soll gemäß Art. 20 der Geschlechtswechsel für die Anwendbarkeit irrelevant sein. Verkannt wird von solchen Normen, dass das Ausüben und Erleiden sexualisierter Gewalt unabhängig von dem Geschlecht eines Individuums geschehen kann.

Zweigeschlechtlichkeit wird zudem dadurch normalisiert, indem die Anerkennung des gelebten Geschlechts des transgeschlechtlichen Subjekts um den Preis der Pathologisierung erfolgt. Das Statut bestimmt in Art. 2 (I) (a), dass das Gender Recognition Panel ${ }^{21}$ einen Antrag annehmen muss, wenn das antragstellende Individuum u.a. eine Geschlechtsidentitätsstörung hat oder hatte. Im Gegensatz zu frühen Gesetzen zum Geschlechtswechsel, die sich lediglich auf >Transsexualismus ${ }^{22}$ bezogen, weitet das Gesetz im Vereinigten Königreich den Einzugsbereich der zu regulierenden Subjekte mit dem breiten medizinisch-psychiatrischen Begriff der Geschlechtsidentitätsstörungen (gender dysphoria) ${ }^{23}$ aus.

Hierbei wird die Geschlechtsbestimmung medizinisch reguliert. ${ }^{24}$ Art. 3 (I) (a) und (b) fordern, dass wahlweise zwei ärztliche oder ein ärztlicher und ein psychologischer Bericht die Diagnose der >Geschlechtsidentitätsstörungen< festhält. In beiden Kombinationen muss nur eine_r der Berichterstatter_innen auf dem Gebiet der >Störungen der Geschlechtsidentität< kundig sein, wobei es sich bei der zweiten Möglichkeit um eine_n Psychologin/Psychologen handeln muss.

20 Die Verantwortung für die Dauerhaftigkeit dieses Bestrebens obliegt somit nicht wie im deutschen Transsexuellengesetz (TSG) den Sachverständigen, sondern dem antragstellenden Individuum. Die Dauerhaftigkeit ist im GRA rigoroser reguliert als im TSG. So ist gemäß \ I Abs. I Nr. 2 TSG einem Antrag stattzugeben, wenn u.a. gutachterlich bestätigt worden ist, dass sich die Geschlechtszugehörigkeit »mit hoher Wahrscheinlichkeit « nicht mehr ändern wird. Gesetz über die Änderung der Vornamen und die Feststellung der Geschlechtszugehörigkeit in besonderen Fällen (Transsexuellengesetz - TSG) BGBl. I980, I654.

2I Anders als es das TSG in $\$ 2$ Abs. I Nr. I vorsieht, obliegt die Anerkennung des Geschlechts im Vereinigten Königreich nicht dem Äquivalent eines Amtsgerichts, sondern gemäß Art. 4 (I) dem Gender Recognition Panel. Dieses setzt sich zusammen aus medizinischen und juristischen Fachleuten. Vgl. Office of Public Sector Information, Explanatory Notes to Gender Recognition Act, 2004, Nr. I-76, Nr. I I; I3, [www.opsi.gov.uk/acts/en2004/2004eno7.htm].

22 Transsexualismus, der unter die Klassifikation der Geschlechtsidentitätsstörungen gefasst wird, wird international definiert als »[d]er Wunsch, als Angehöriger des anderen Geschlechts zu leben und anerkannt zu werden. Dieser geht meist mit Unbehagen oder dem Gefühl der Nichtzugehörigkeit zum eigenen anatomischen Geschlecht einher. Es besteht der Wunsch nach chirurgischer und hormoneller Behandlung, um den eigenen Körper dem bevorzugten Geschlecht soweit wie möglich anzugleichen. « Deutsches Institut für Medizinische Dokumentation und Information, ICD-ı, Internationale Klassifikation der Krankheiten. GM-Version 2008, F.64.0, [www.dimdi.de/static/de/klassi/diagnosen/icd Io/htmelgm2008/fr-icd.htm].

23 Das medizinisch-psychiatrische Konzept der Geschlechtsidentitätsstörungen umfasst nach den Standards der »Harry Benjamin International Gender Dysphoria Association« Individuen, die in unterschiedlichem Maße persönliche Unzufriedenheit u.a. mit der sexuellen Identität, der Geschlechtsidentität, geschlechtlichen Zuschreibungen, vergeschlechtlichter morphologischer Beschaffenheit und der Geschlechterrolle aufweisen. Vgl. Meyer et al. (Fn. 8), 2.

24 Allerdings ist die Geschlechtsbestimmung auch bei konventionell genuisierten Geschlechtern nicht selbstbestimmt. Vielmehr wird jedem Individuum ein Geschlecht zugewiesen, dessen Normen es zu wiederholen hat. 
Die Normalisierung und Naturalisierung von Zweigeschlechtlichkeit spiegelt sich ferner wider in der Verwendung des Begriffs des serworbenen< (acquired) Geschlechts bei transgeschlechtlichen Individuen. Die einseitige Zuschreibung von Transgeschlechtern als >erworben $<$ verschleiert den Konstruktionscharakter aller Geschlechter. ${ }^{25}$

Zugleich ist eine Aufweichung des vermeintlich natürlichen Zusammenhangs von Morphologie und Geschlecht zu verzeichnen. Im Gegensatz zum TSG, das gemäß $\int 8$ Abs. I Nr. 3 und 4 zur Anerkennung des empfundenen Geschlechts einer transgeschlechtlichen Person die dauerhafte Fortpflanzung und einen geschlechtsangleichenden operativen Eingriff voraussetzt, fordert der GRA keinerlei medizinische oder chirurgische Maßnahmen. ${ }^{26}$ Demnach kann ein weiblicher Körper ebenso einen Mann wie eine Frau darstellen sowie ein männlicher Körper eine Frau oder einen Mann.

Dennoch bestätigt das Gesetz heteronormative Zweigeschlechtlichkeit, indem es - im Einklang mit Art. I Ic des Matrimonial Causes Act - die Ehe als exklusives Rechtsinstitut verschiedengeschlechtlicher Paare widerspiegelt. Für verheiratete Transpersonen ist nur eine Interimsversion der Anerkennung (interim gender recognition certificate) vorgesehen. ${ }^{27}$ Erst nach einer Annullierung oder Auflösung der Ehe, die nach Art. 5 (2) (a) innerhalb von sechs Monaten nach der Ausstellung der Interimsurkunde erfolgen muss, kann nach Art. 5 (2) eine volle Anerkennungsurkunde (full gender recognition certificate) beantragt werden.

Der im gleichen Jahr wie der GRA verabschiedete Civil Partnership Act (2004) bietet gleichgeschlechtlichen Paaren zwar ein im Hinblick auf Rechte und Pflichten nahezu gleichberechtigtes Rechtsinstitut. ${ }^{28}$ Nach Büchler ist es aber gerade die Aufrechterhaltung der Ehe als einem verschiedengeschlechtlichen Institut, mit der Zweigeschlechtlichkeit reproduziert und die Geschlechterdifferenz inszeniert wird. ${ }^{29}$

\section{Fazit}

Eine queere Kritik des Rechts richtet die Aufmerksamkeit auf die Reproduktion und Normalisierung von komplex strukturierten sozialen Ungleichheitsverhältnissen in Gesetzen, Rechtsprechung und Rechtsinstituten. Insbesondere betrachtet queer theory kritisch und mit dekonstruktivistischer Ausrichtung, wie das Recht dabei mitwirkt, die sozialen Konstrukte Zweigeschlechtlichkeit und Heterosexualität derart zu normalisieren und in historisch-spezifischer Weise zu wiederholen, dass sie naturhaft erscheinen. Im dargelegten Beispiel geschieht dies durch die Verschleierung des Konstruktionscharakters polarisierter Männlichkeit und Weiblichkeit, der Herstellung, Pathologisierung und Regulierung minorisierter Subjekte sowie durch die Produktion erneuter Ausschlüsse derer, die den rechtlichen Regeln der Vergeschlechtlichung nicht folgen.

25 Vgl. Gesa Lindemann, Das paradoxe Geschlecht. Transsexualität im Spannungsfeld von Körper, Leib und Gefühlen, Frankfurt am Main I993, I I.

26 Sie bleiben aber unabhängig vom Gesetz weiterhin eine Option.

$27 \$ 8$ Abs. (I) Nr. 4 TSG setzt hingegen bereits bei Antragstellung zur Personenstandsänderung voraus, dass das betreffende Individuum nicht verheiratet ist.

28 S. hierzu Civil Partnership Act (2004), [www.opsi.gov.uk/acts/acts2004/ukpga_20040033_en_r]. Der deutsche Gesetzgeber hingegen hat die Eingetragene Partnerschaft von 200 I als deutlich niederrangig zur Ehe gestaltet.

29 Andrea Büchler, Eherecht und Geschlechterkonstruktion. Ein Beitrag zur Abschaffung der institutionalisierten Zweigeschlechtlichkeit, in: Verein ProFri - Schweizer Feministisches Rechtsinstitut (Hrsg.), UT: Beiträge zur feministischen Rechtswissenschaft, Lachen/ St. Gallen 200I, 59-90, 62, 76. 